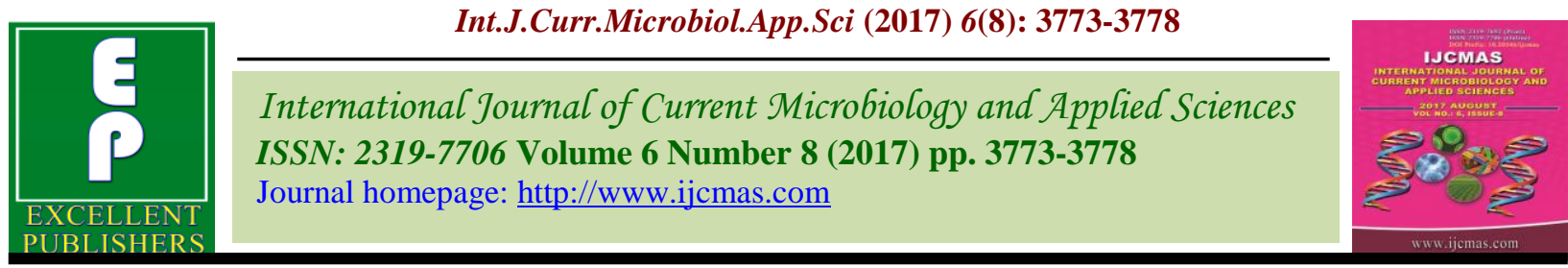

Original Research Article

https://doi.org/10.20546/ijcmas.2017.608.456

\title{
Effect of Quinchlorac on Grassy Weeds in Transplanted Rice
}

\author{
Y.M. Ramesha ${ }^{1 *}$, Manjunatha Bhanuvally², Ashok Kumar Gaddi², \\ D. Krishamurthy ${ }^{1}$ and M.R. Umesh ${ }^{1}$ \\ ${ }^{1}$ Department of Agronomy, University of Agricultural Sciences, Raichur, Karnataka, India \\ ${ }^{2}$ Department of Soil Science and Agricultural Chemistry, University of Agricultural Sciences, \\ Raichur, Karnataka, India \\ *Corresponding author
}

A B S T R A C T

Keywords

Weed dry weight,

Weed control

efficiency,

Grain yield, Rice.

Article Info

Accepted:

21 June 2017

Available Online:

10 August 2017
An experiment was conducted during Kharif 2015 and 2016, at Agricultural Research Station, Dhadesugur, University of Agricultural Sciences, Raichur, Karnataka, India, to study the effect of Quinchlorac on control of grassy weeds and productivity of transplanted rice in Northern dry zone of Karnataka. Sprays of Quinchlorac $250 \mathrm{~g} / 1 \mathrm{SC} @ 125 \mathrm{~g}$ a.i. /ha, Quinchlorac 250 g/l SC @ $187.5 \mathrm{~g}$ a.i. /ha, Quinchlorac 250 g/l SC @ $250 \mathrm{~g}$ a.i. /ha, Quinchlorac 250 g/1 SC @ 312.5 g a.i. /ha, Cyhalofop butyl $10 \%$ EC @ $100 \mathrm{~g}$ a.i. /ha, Penoxsulam $21.7 \%$ SC @ $20 \mathrm{~g}$ a.i./ha, Azimsulfuron 50\% DF @ $28 \mathrm{~g}$ a.i./ha, hand weeding at 15 and 40 days after planting (weed free check) and a weedy check (untreated check) were also maintained. A result revealed that, application of Quinchlorac $250 \mathrm{~g} / \mathrm{l} \mathrm{SC} @ 250 \mathrm{~g}$ a.i/ha was most effective in controlling the grassy weeds and increases the grain yield of transplanted rice without any phytotoxic effect.

\section{Introduction}

Rice has been staple food for more than 60 per cent of the world population, providing energy for about $40 \%$ of the world population where every third person on earth consumes rice every day in one form or other (Datta and Khushi, 2002). Therefore, crop paddy (Oryza sativa L.) is an important crop which is extensively grown in tropical and subtropical regions of the world. There are several reasons for its low productivity but the losses due to weeds are one of the most important. More than one third of the total loss $(33 \%)$ is caused by weeds alone (Verma et al., 2015). Weeds are most severe and widespread biological constraints to crop production in India. Weeds are responsible for heavy yield losses in paddy, to the extent of complete crop failure under severe infestation conditions. Irrespective of the method of paddy establishment, weeds are a major impediment to paddy production due to their ability to compete for resources. In general, weeds problem in transplanted paddy is lower than that of direct seeded paddy because of puddling and stagnation of water in transplanted paddy during early growth stage of crop. But in some cases where continuous standing water cannot be maintained 
particularly for the first 45 days, weed infestation in transplanted paddy also may be as high as direct seeded paddy. According to Singh et al., (2004) weeds can reduce the grain yield of dry-seeded paddy (DSR) by $75.8 \%$, wet seeded paddy (WSR) by $70.6 \%$ and transplanted paddy (TPR) by $62.6 \%$. Weeds by virtue of their high adaptability and faster growth dominate the crop habitat and reduce the yield potential. Therefore, the present investigation was undertaken to study the effect of early post emergent herbicide for control of major grassy weeds in transplanted rice.

\section{Materials and Methods}

An experiment was conducted during Kharif 2015 and 2016 on effect of Quinchlorac 250 $\mathrm{g} / \mathrm{l} \mathrm{SC}$ on grassy weeds in transplanted rice at Agricultural Research Station, Dhadesugur, Karnataka, India. The soil of the experimental site was deep black and neutral in $\mathrm{pH}$ (8.04), EC $(0.47 \mathrm{ds} / \mathrm{m})$, medium in organic carbon content $(0.41 \%)$, low in nitrogen $(224 \mathrm{~kg} / \mathrm{ha})$, medium in phosphorus $(58.5 \mathrm{~kg} / \mathrm{ha})$ and potassium $(428.5 \mathrm{~kg} / \mathrm{ha})$. The total actual rainfall received during 2015 and 2016 were 642.3 and $655.6 \mathrm{~mm}$, respectively. It was almost good as compared to the average normal rainfall received over the last thirty years. The overall pest and disease incidence was least during this season. There are nine treatments comprising of Quinchlorac at different concentrations, Cyhalofop butyl 10 \% EC @ 100 g a.i. /ha, Penoxsulam 21.7\% SC @ $20 \mathrm{~g}$ a.i./ha, Azimsulfuron 50\% DF @ $28 \mathrm{~g}$ a.i./ha, hand weeding at 15 and 40 days after planting (weed free check) and a weedy check (untreated check) and replicated thrice. The randomized block design was adopted for this experiment. Twenty five days old age seedlings were planted and herbicide was sprayed as per the treatments. On pre-spray, 30 and 60 days after transplanting (DAT) and total dry weight of grassy weeds $\left(\mathrm{g} / \mathrm{m}^{2}\right)$ were taken in both treated and untreated plots. Species wise, weed population were recorded at before spray, 30 and 60 DAT using quadrates of $1.0 \mathrm{~m}^{2}$. Further, total dry weight grassy weeds were recorded and used for calculating weed control efficiency (WCE). Data on total dry weight of weeds were analysed statistically. Weed control efficiency (WCE) was calculated as follows. WCE=Dry weight of weeds under control plot-Dry weight of weeds under treatments/Dry weight of weeds under control plot X 100. Five plants were randomly selected in each plot of each replication and were tagged for the purpose of recording observations on number of filled grains per panicle.

Similarly, grain and straw from each net plot in each replication was harvested and dried. The grains after threshing were weighed and recorded as grain yield per net plot. Further, this net plot grain yield was converted to grain yield per hectare. The data were analyzed as per the procedure of Gomez and Gomez, 1984.

\section{Results and Discussion}

\section{Weed flora of experimental site}

In the experimental plots, the dominant grassy weeds were Echinochloa sp, Panicum repens, Cynodon doctylon, Leptochloa chinensis and Bracharia sp. etc.

\section{Dry weight of grassy weeds}

Results revealed that, Hand weeding at 15 and 40 days after transplanting resulted in zero dry weight means weed free was maintained throughout the crop period. Further, significant reduction in dry weight of grassy weeds per square meter was observed under all the doses of Quinchlorac $250 \mathrm{~g} / \mathrm{l} \mathrm{SC}$ application compared to untreated control and other herbicide application treatments. 
Table.1 Effect of weed control treatments on dry weight of grassy weeds in transplanted rice

\begin{tabular}{|c|c|c|c|c|c|c|c|c|c|}
\hline \multirow{3}{*}{ Treatments } & \multicolumn{9}{|c|}{ Dry weight of grassy weeds $\left(\mathrm{g} / \mathrm{m}^{2}\right)$} \\
\hline & \multicolumn{3}{|c|}{ Before spraying } & \multicolumn{3}{|c|}{30 DAT } & \multicolumn{3}{|c|}{60 DAT } \\
\hline & 2015 & 2016 & Pooled & 2015 & 2016 & Pooled & 2015 & 2016 & Pooled \\
\hline $\mathbf{T}_{1}$ : Quinchlorac $250 \mathrm{~g} / 1 \mathrm{SC} @ 125 \mathrm{~g}$ a.i/ha & $\begin{array}{c}4.36 \\
(18.0)\end{array}$ & $\begin{array}{c}4.36 \\
(18.0)\end{array}$ & $\begin{array}{c}4.36 \\
(18.0)\end{array}$ & $\begin{array}{c}3.66 \\
(12.4)\end{array}$ & $\begin{array}{c}3.68 \\
(12.5)\end{array}$ & $\begin{array}{c}3.67 \\
(12.5)\end{array}$ & $\begin{array}{c}4.42 \\
(18.5)\end{array}$ & $\begin{array}{c}4.46 \\
(18.9)\end{array}$ & $\begin{array}{c}4.44 \\
(18.7)\end{array}$ \\
\hline $\mathbf{T}_{2}:$ Quinchlorac $250 \mathrm{~g} / 1$ SC @ $187.5 \mathrm{~g}$ a.i/ha & $\begin{array}{c}4.38 \\
(18.2) \\
\end{array}$ & $\begin{array}{c}4.34 \\
(17.8) \\
\end{array}$ & $\begin{array}{c}4.36 \\
(18.0) \\
\end{array}$ & $\begin{array}{c}3.40 \\
(10.5) \\
\end{array}$ & $\begin{array}{c}3.40 \\
(10.6) \\
\end{array}$ & $\begin{array}{c}3.40 \\
(10.6) \\
\end{array}$ & $\begin{array}{c}3.92 \\
(14.4) \\
\end{array}$ & $\begin{array}{c}3.97 \\
(14.8) \\
\end{array}$ & $\begin{array}{c}3.95 \\
(14.6) \\
\end{array}$ \\
\hline $\mathbf{T}_{3}$ : Quinchlorac $250 \mathrm{~g} / 1 \mathrm{SC} @ 250 \mathrm{~g}$ a.i/ha & $\begin{array}{c}4.35 \\
(17.9)\end{array}$ & $\begin{array}{c}4.35 \\
(17.9) \\
\end{array}$ & $\begin{array}{c}4.35 \\
(17.9) \\
\end{array}$ & $\begin{array}{c}3.34 \\
(10.1)\end{array}$ & $\begin{array}{c}3.36 \\
(10.3)\end{array}$ & $\begin{array}{c}3.35 \\
(10.2) \\
\end{array}$ & $\begin{array}{c}3.66 \\
(12.4) \\
\end{array}$ & $\begin{array}{c}3.81 \\
(13.5) \\
\end{array}$ & $\begin{array}{c}3.73 \\
(13.0) \\
\end{array}$ \\
\hline $\mathbf{T}_{4}$ : Quinchlorac $250 \mathrm{~g} / 1$ SC @ $312.5 \mathrm{~g}$ a.i/ha & $\begin{array}{c}4.38 \\
(18.2) \\
\end{array}$ & $\begin{array}{c}4.41 \\
(18.5) \\
\end{array}$ & $\begin{array}{c}4.40 \\
(18.4) \\
\end{array}$ & $\begin{array}{l}3.22 \\
(9.4) \\
\end{array}$ & $\begin{array}{c}3.36 \\
(10.3) \\
\end{array}$ & $\begin{array}{l}3.29 \\
(9.8) \\
\end{array}$ & $\begin{array}{c}3.39 \\
(10.5) \\
\end{array}$ & $\begin{array}{c}3.44 \\
(10.8) \\
\end{array}$ & $\begin{array}{c}3.41 \\
(10.7) \\
\end{array}$ \\
\hline $\mathbf{T}_{5}:$ Cyhalofop butyl $10 \%$ EC @ 100 g a. i. /ha & $\begin{array}{c}4.39 \\
(18.3) \\
\end{array}$ & $\begin{array}{c}4.39 \\
(18.3) \\
\end{array}$ & $\begin{array}{c}4.39 \\
(18.3) \\
\end{array}$ & $\begin{array}{c}4.04 \\
(15.3) \\
\end{array}$ & $\begin{array}{c}4.07 \\
(15.6) \\
\end{array}$ & $\begin{array}{c}4.06 \\
(15.5) \\
\end{array}$ & $\begin{array}{c}5.10 \\
(25.0) \\
\end{array}$ & $\begin{array}{c}5.12 \\
(25.2) \\
\end{array}$ & $\begin{array}{c}5.11 \\
(25.1) \\
\end{array}$ \\
\hline $\mathbf{T}_{6}:$ Penoxsulam $21.7 \%$ SC @ 20 g a.i./ha & $\begin{array}{c}4.38 \\
(18.2) \\
\end{array}$ & $\begin{array}{c}4.38 \\
(18.2) \\
\end{array}$ & $\begin{array}{c}4.38 \\
(18.2) \\
\end{array}$ & $\begin{array}{c}3.95 \\
(14.6) \\
\end{array}$ & $\begin{array}{c}3.95 \\
(14.6) \\
\end{array}$ & $\begin{array}{c}3.95 \\
(14.6) \\
\end{array}$ & $\begin{array}{c}4.84 \\
(22.4) \\
\end{array}$ & $\begin{array}{c}4.90 \\
(23.0) \\
\end{array}$ & $\begin{array}{c}4.87 \\
(22.7) \\
\end{array}$ \\
\hline $\mathbf{T}_{7}:$ Azimsulfuron 50\% DF @ $28 \mathrm{~g}$ a.i./ha & $\begin{array}{c}4.44 \\
(18.7) \\
\end{array}$ & $\begin{array}{c}4.44 \\
(18.7) \\
\end{array}$ & $\begin{array}{c}4.44 \\
(18.7) \\
\end{array}$ & $\begin{array}{c}4.23 \\
(16.9) \\
\end{array}$ & $\begin{array}{c}4.24 \\
(17.0) \\
\end{array}$ & $\begin{array}{c}4.23 \\
(16.9) \\
\end{array}$ & $\begin{array}{c}5.29 \\
(27.0) \\
\end{array}$ & $\begin{array}{c}5.31 \\
(27.2) \\
\end{array}$ & $\begin{array}{c}5.30 \\
(27.1) \\
\end{array}$ \\
\hline $\mathbf{T}_{\mathbf{8}}$ : Hand weeding & $\begin{array}{l}1.00 \\
(0.0) \\
\end{array}$ & $\begin{array}{l}1.00 \\
(0.0) \\
\end{array}$ & $\begin{array}{l}1.00 \\
(0.0) \\
\end{array}$ & $\begin{array}{c}1.00 \\
(0.00) \\
\end{array}$ & $\begin{array}{c}1.00 \\
(0.00) \\
\end{array}$ & $\begin{array}{c}1.00 \\
(0.00) \\
\end{array}$ & $\begin{array}{l}1.00 \\
(0.0) \\
\end{array}$ & $\begin{array}{l}1.00 \\
(0.0) \\
\end{array}$ & $\begin{array}{l}1.00 \\
(0.0) \\
\end{array}$ \\
\hline $\mathbf{T}_{\mathbf{9}}:$ Weedy check & $\begin{array}{c}4.49 \\
(19.2) \\
\end{array}$ & $\begin{array}{c}4.53 \\
(19.5) \\
\end{array}$ & $\begin{array}{c}4.51 \\
(19.4) \\
\end{array}$ & $\begin{array}{c}8.23 \\
(66.8) \\
\end{array}$ & $\begin{array}{c}8.29 \\
(67.8) \\
\end{array}$ & $\begin{array}{c}8.26 \\
(67.3) \\
\end{array}$ & $\begin{array}{c}10.3 \\
(105.2) \\
\end{array}$ & $\begin{array}{c}10.5 \\
(109.2) \\
\end{array}$ & $\begin{array}{c}10.4 \\
(107.2) \\
\end{array}$ \\
\hline C.D@ 5\% & 2.56 & 2.13 & 2.41 & 2.12 & 2.52 & 2.01 & 4.56 & 6.52 & 5.14 \\
\hline
\end{tabular}

Note: Figures in the parenthesis are square root transformed values (sq. root of $\mathrm{x}+1$ ), DAT- Days after transplanting, SC- Suspension Concentrates, EC-

Emulsifiable Concentrates, DF- Dry Flowable 
Table. 2 Effect of weed control treatments on Weed control efficiency of grasses in transplanted rice

\begin{tabular}{|c|c|c|c|c|c|c|c|c|c|}
\hline \multirow{3}{*}{ Treatments } & \multicolumn{9}{|c|}{ Weed control efficiency $(\%)$} \\
\hline & \multicolumn{3}{|c|}{ Before spraying } & \multicolumn{3}{|c|}{ 30 DAT } & \multicolumn{3}{|c|}{60 DAT } \\
\hline & 2015 & 2016 & Pooled & 2015 & 2016 & Pooled & 2015 & 2016 & Pooled \\
\hline $\mathbf{T}_{1}$ : Quinchlorac 250 g/l SC @ $125 \mathrm{~g}$ a.i/ha & 6.25 & 7.64 & 6.95 & 81.4 & 81.5 & 81.5 & 82.4 & 82.7 & 82.6 \\
\hline $\mathbf{T}_{2}$ : Quinchlorac $250 \mathrm{~g} / 1 \mathrm{SC} @ 187.5 \mathrm{~g}$ a.i/ha & 5.36 & 8.56 & 6.96 & 84.2 & 84.4 & 84.3 & 86.3 & 86.4 & 86.4 \\
\hline $\mathbf{T}_{3}:$ Quinchlorac $250 \mathrm{~g} / 1 \mathrm{SC} @ 250 \mathrm{~g}$ a.i/ha & 6.61 & 8.00 & 7.31 & 84.8 & 84.8 & 84.8 & 88.2 & 87.7 & 87.9 \\
\hline $\mathbf{T}_{4}$ : Quinchlorac $250 \mathrm{~g} / 1 \mathrm{SC} @ 312.5 \mathrm{~g}$ a.i/ha & 5.10 & 5.23 & 5.17 & 86.0 & 84.9 & 85.4 & 90.0 & 90.1 & 90.1 \\
\hline T $_{5}:$ Cyhalofop butyl $10 \%$ EC @ 100 g a. i. /ha & 4.69 & 6.41 & 5.55 & 77.1 & 77.0 & 77.0 & 76.2 & 76.9 & 76.6 \\
\hline $\mathbf{T}_{6}:$ Penoxsulam $21.7 \%$ SC @ 20 g a.i./ha & 5.21 & 6.67 & 5.94 & 78.2 & 78.4 & 78.3 & 78.7 & 78.9 & 78.8 \\
\hline $\mathbf{T}_{7}$ : Azimsulfuron 50\% DF @ $28 \mathrm{~g}$ a.i./ha & 2.55 & 3.90 & 3.22 & 74.7 & 75.0 & 74.8 & 74.3 & 75.1 & 74.7 \\
\hline $\mathbf{T}_{\mathbf{8}}:$ Hand weeding & 100 & 100 & 100 & 100 & 100 & 100 & 100 & 100 & 100 \\
\hline $\mathbf{T}_{\mathbf{9}}$ : Weedy check & - & - & - & - & - & - & - & - & - \\
\hline C.D@ 5\% & 5.12 & 4.12 & 4.25 & 1.05 & 1.06 & 1.02 & 3.10 & 3.24 & 3.21 \\
\hline
\end{tabular}

Note: Figures in the parenthesis are square root transformed values (sq. root of $\mathrm{x}+1$ ), DAT- Days after transplanting, SC- Suspension Concentrates, ECEmulsifiable Concentrates, DF- Dry Flowable

Table.3 Effect of weed control treatments on productivity of transplanted rice

\begin{tabular}{|c|c|c|c|c|c|c|c|c|c|}
\hline \multirow{2}{*}{ Treatments } & \multicolumn{3}{|c|}{ Number of filled grains per panicle } & \multicolumn{3}{|c|}{ Grain vield (kg/ha) } & \multicolumn{3}{|c|}{ Straw yield (kg/ha) } \\
\hline & 2015 & 2016 & Pooled & 2015 & 2016 & Pooled & 2015 & 2016 & Pooled \\
\hline $\mathbf{T}_{1}$ : Quinchlorac $250 \mathrm{~g} / 1 \mathrm{SC} @ 125 \mathrm{~g}$ a.i/ha & 173 & 175 & 174 & 4340 & 4620 & 4480 & 4690 & 5082 & 4886 \\
\hline $\mathbf{T}_{2}:$ Quinchlorac $250 \mathrm{~g} / 1 \mathrm{SC} @ 187.5 \mathrm{~g}$ a.i/ha & 175 & 176 & 175 & 5208 & 5421 & 5315 & 5655 & 5963 & 5809 \\
\hline $\mathbf{T}_{3}:$ Quinchlorac $250 \mathrm{~g} / 1 \mathrm{SC} @ 250 \mathrm{~g}$ a.i/ha & 179 & 181 & 180 & 5324 & 5524 & 5424 & 5895 & 6076 & 5986 \\
\hline $\mathbf{T}_{4}:$ Quinchlorac 250 g/1 SC @ $312.5 \mathrm{~g}$ a.i/ha & 185 & 186 & 186 & 5440 & 5623 & 5531 & 5924 & 6185 & 6055 \\
\hline T $_{5}:$ Cyhalofop butyl $10 \%$ EC @ 100 g a. i. /ha & 174 & 176 & 175 & 4803 & 4985 & 4894 & 5209 & 5484 & 5346 \\
\hline $\mathbf{T}_{6}:$ Penoxsulam $21.7 \%$ SC @ $20 \mathrm{~g}$ a.i./ha & 172 & 174 & 173 & 5093 & 5124 & 5108 & 5649 & 5636 & 5643 \\
\hline $\mathbf{T}_{7}$ : Azimsulfuron 50\% DF @ $28 \mathrm{~g}$ a.i./ha & 172 & 175 & 174 & 4688 & 4765 & 4726 & 5072 & 5242 & 5157 \\
\hline $\mathbf{T}_{\mathbf{8}}:$ Hand weeding & 187 & 189 & 188 & 5903 & 5988 & 5945 & 6407 & 6587 & 6497 \\
\hline $\mathbf{T}_{\mathbf{9}}:$ Weedy check & 172 & 174 & 173 & 3472 & 3456 & 3464 & 3759 & 3802 & 3780 \\
\hline C.D@ 5\% & 5.82 & 6.25 & 7.26 & 562.3 & 462.1 & 526.2 & 652.1 & 504.0 & 505.2 \\
\hline
\end{tabular}

Note: Figures in the parenthesis are square root transformed values (sq. root of $\mathrm{x}+1$ ), DAT- Days after transplanting, SC- Suspension Concentrates, EC-

Emulsifiable Concentrates, DF- Dry Flowable 
Table.4 Economics of transplanted rice as influenced by weed control treatments (Mean data 2015 and 2016)

\begin{tabular}{|l|c|c|c|c|}
\hline \multicolumn{1}{|c|}{ Treatments } & $\begin{array}{c}\text { Cost of cultivation } \\
\text { (Rs/ha) }\end{array}$ & $\begin{array}{c}\text { Gross returns } \\
\text { (Rs/ha) }\end{array}$ & $\begin{array}{c}\text { Net returns } \\
\text { (Rs/ha) }\end{array}$ & $\begin{array}{c}\text { B:C } \\
\text { ratio }\end{array}$ \\
\hline $\mathbf{T}_{\mathbf{1}}$ : Quinchlorac 250 g/1 SC @ 125 g a.i/ha & 30635 & 80852 & 50217 & 2.64 \\
\hline $\mathbf{T}_{\mathbf{2}}$ : Quinchlorac 250 g/1 SC @ 187.5 g a.i/ha & 30735 & 96004 & 65269 & 3.12 \\
\hline $\mathbf{T}_{\mathbf{3}}$ : Quinchlorac 250 g/1 SC @ 250 g a.i/ha & 30835 & 98104 & 67269 & 3.18 \\
\hline $\mathbf{T}_{\mathbf{4}}$ : Quinchlorac 250 g/1 SC @ 312.5 g a.i/ha & 30935 & 99958 & 69023 & 3.23 \\
\hline $\mathbf{T}_{\mathbf{5}}:$ Cyhalofop butyl 10 \% EC @ 100 g a. i. /ha & 31935 & 88409 & 56474 & 2.77 \\
\hline $\mathbf{T}_{\mathbf{6}}$ : Penoxsulam 21.7 \% SC @ 20 g a.i./ha & 32147 & 92491 & 60343 & 2.87 \\
\hline $\mathbf{T}_{\mathbf{7}}$ : Azimsulfuron 50\% DF @ 28 g a.i./ha & 30547 & 85418 & 54871 & 2.80 \\
\hline $\mathbf{T}_{\mathbf{8}}$ : Hand weeding & 35435 & 107478 & 72043 & 3.03 \\
\hline $\mathbf{T}_{\mathbf{9}}$ : Weedy check & 30435 & 62649 & 32214 & 2.06 \\
\hline & NA & NA & $\mathbf{4 6 5 2 . 2}$ & $\mathbf{0 . 1 8}$ \\
\hline
\end{tabular}

Note: NA: Not Analyzed, SC- Suspension Concentrates, EC- Emulsifiable Concentrates, DF- Dry Flowable,Urea $@$ Rs. $5.00 \mathrm{~kg}^{-1}$, DAP @Rs. $20.00 \mathrm{~kg}^{-1}$, MoP @Rs. $15.00 \mathrm{~kg}^{-1}$, Quinchlorac $250 \mathrm{~g} / 1 \mathrm{SC} @$ Rs.400 l-1, Penoxsulum $21.7 \%$ SC @ Rs.1712 ha ${ }^{-1}$, Clincher 10\% EC @ Rs.1500 ha ${ }^{-1}$,Azimsulfuron 50\% DF @ Rs.2000 ha ${ }^{-1}$, Grain @ Rs $17.0 \mathrm{~kg}^{-1}$ and Straw @ Rs $1.0 \mathrm{~kg}^{-1}$.

Among weed control treatments, application of Quinchlorac $250 \mathrm{~g} / 1$ SC @ $312.5 \mathrm{~g}$ a.i./ha recorded significantly lower dry weight of grassy weeds at 30 and 60 days after transplanting and which was onpar with the application of Quinchlorac $250 \mathrm{~g} / \mathrm{l} \mathrm{SC} @ 250 \mathrm{~g}$ a.i./ha and Quinchlorac $250 \mathrm{~g} / 1$ SC @ 187.5g a.i./ha compared to other weed control treatments in both the years.

These results are conformity with the findings of Abeysekera (1999) stated that, application of tank mixture of quichlorac @ $50 \mathrm{~g} / \mathrm{ha}+$ propanil @ 1.08 kg/ha controlled effectively the grassy weeds and recorded lower dry weight in wet seeded rice in mid country region of Srilanka. Whereas, higher dry weight of grassy weeds was observed in weedy check treatment. This might be due high weed infestation (Table 1).

\section{Weed control efficiency}

In both the years, Cent per cent weed control efficiency was noticed in hand weeding treatment because weed free maintained in throughout the crop period. Further, significantly higher weed control efficiency was observed under all the doses of Quinchlorac 250 g/l SC application treatments compared to untreated control and other herbicide application treatments. Among weed control treatments, application of Quinchlorac $250 \mathrm{~g} / \mathrm{l}$ SC @ $312.5 \mathrm{~g}$ a.i./ha recorded significantly higher weed control efficiency at 30 and 60 days after transplanting and which was onpar with the application of Quinchlorac $250 \mathrm{~g} / \mathrm{l} \mathrm{SC}$ @ 250g a.i./ha and Quinchlorac 250 g/1 SC @ $187.5 \mathrm{~g}$ a.i./ha compared to other weed control treatments. These results are conformity with the findings of Amarasinghe et al., (1999) stated that, application of quichlorac @ 500 $\mathrm{g} / \mathrm{ha}$ recorded higher weed control efficiency in wet seeded rice in mid country region of Srilanka.

Similarly, lower weed control efficiency was noticed in weedy check treatment (Table 2).

\section{Yield components and yield of rice}

The number of filled grains per panicle was significantly higher in hand weeded treatment and lower in un-weeded treatment in both the years. Similarly, significantly higher grain and straw yield were observed in weed free treatment and which was on par with the application of Quinchlorac 250 g/l SC @ 312.5 
g a.i./ha, Quinchlorac 250 g/l SC @ 250g a.i./ha and Quinchlorac 250 g/l SC @ 187.5g a.i./ha compared to other weed control treatments. These results are conformity with the findings of Abeysekera (1999) stated that, application of tank mixture of quichlorac @ 50 g/ha + propanil@1.08 kg/ha controlled effectively the grassy weeds in wet seeded rice and resulted in higher grain yield in mid country region of Srilanka. Similar results also reported by Amarasinghe et al., (1999). Whereas, lower grain and straw yield were recorded in weedy check plot. This is due to the higher infestation of weeds Seema et al., (2015) also stated that, higher grain yield of aerobic rice was recorded in weed control treatments over the un-weeded treatment (Table 3).

\section{Effect of weed control treatments on} economics

Mean data of 2015 and 2016 showed that, application of Quinchlorac 250 g/l SC @ 312.5 $\mathrm{g}$ a.i./ha recorded significantly higher benefit cost ratio and which was on par with the application of Quinchlorac 250 g/l SC @ 250g a.i./ha and Quinchlorac $250 \mathrm{~g} / \mathrm{l} \mathrm{SC}$ @ 187.5g a.i./ha compared to other weed control treatments. This might be due higher grain and straw yield and less cost of establishment. Further, lower benefit cost ratio was noticed in weedy check treatment where weed infestation was more and resulting in lower grain and straw yield (Table 4). Similar finding were also reported by Amarasinghe and Marambe (1998).

The results indicated that, application of Quinchlorac 250 g/l SC @ 187.5g a.i/ha or 250 g.a.i/ha was found to be more effective in control of grassy weeds over other weed control treatments without showing any phytotoxic symptoms to the rice plants.

\section{References}

Abeysekara, A., 1999. Current status of weed control in rice in Srilanka. Proceedings of the $17^{\text {th }}$ Asian Pacific Weed Science Conference, 22-27 November. Thailand. p 174-18.

Amarasinghe, L., and B. Marambe, 1998. Trends in weed control of rice cultivation in Srilanka. Proceedings of multidisciplinary International Conference. University of Peradeniya, Srilanka. pp. 112 (supplement)

Amarasinghe, L., B. Marambe and R.P.A.D. Rajpakse, 1999. Effect of Quinchlorac on weed control and productivity of wet seeded rice in the mid region of Sri Lanka. Sri Lankan Journal of Agricultural Sciences 36:24-34

Datta, S.K., and Khushi, G.S. 2002. Improving rice to meet food and nutrient needs: Biotechnological approaches. J. Crop Production, 6: 229-247.

Gomez, K.A., and A.A. Gomez, 1984. Statistical procedures for agricultural research (2 Ed.). John wiley and sons, New York, 680p

Seema Krishna, M., and Devi, M. T. T. 2014. Effect of nitrogen and weed management on nutrient uptake by weeds under direct seeded aerobic rice. The Bioscan. 9(2): 535-537.

Singh, V.P., Singh G and Singh M. 2004. Effect of fenoxaprop-p-ethyl on transplanted rice and associated weeds. Indian Journal of Weed Science 36(3\&4): 190-192.

Verma, S. K., Singh, S. B., Meena, R. N., Prasad, S. K., Meena R. S. and Gaurav. 2015. A review of weed management in India: The need of new directions for sustainable agriculture. The Bioscan. 10(1): 253-263.

\section{How to cite this article:}

Ramesha, Y. M., Manjunatha Bhanuvally, Ashok Kumar Gaddi, D. Krishamurthy and Umesh, M.R. 2017. Effect of Quinchlorac on Grassy Weeds in Transplanted Rice. Int.J.Curr.Microbiol.App.Sci. 6(8): 3773-3778. doi: https://doi.org/10.20546/ijcmas.2017.608.456 